\title{
New Algorithms for Designing Unimodular Sequences With Good Correlation Properties
}

\author{
Petre Stoica, Fellow, IEEE, Hao He, Student Member, IEEE, and Jian Li, Fellow, IEEE
}

\begin{abstract}
Unimodular (i.e., constant modulus) sequences with good autocorrelation properties are useful in several areas, including communications and radar. The integrated sidelobe level (ISL) of the correlation function is often used to express the goodness of the correlation properties of a given sequence. In this paper, we present several cyclic algorithms for the local minimization of ISL-related metrics. These cyclic algorithms can be initialized with a good existing sequence such as a Golomb sequence, a Frank sequence, or even a (pseudo)random sequence. To illustrate the performance of the proposed algorithms, we present a number of examples, including the design of sequences that have virtually zero autocorrelation sidelobes in a specified lag interval and of long sequences that could hardly be handled by means of other algorithms previously suggested in the literature.
\end{abstract}

Index Terms-Autocorrelation, cyclic algorithms, integrated sidelobe level, merit factor, unimodular sequences, waveform design.

\section{INTRODUCTION AND PROBLEM FORMULATION}

$\mathbf{L}$ ET $\left\{x_{n}\right\}_{n=1}^{N}$ denote the unimodular sequence to be designed. Without introducing any restriction, we can assume that

$$
\left|x_{n}\right|=1, \quad n=1, \ldots, N \text {. }
$$

To keep this paper as concise as possible, we will limit the discussion to general unimodular sequences, but we note here that finite-alphabet unimodular sequences, such as m-ary phase-shift keying sequences, can also be dealt with in our framework-see, however, the remark following (10).

Let

$$
r_{k}=\sum_{n=k+1}^{N} x_{n} x_{n-k}^{*}=r_{-k}^{*}, \quad k=0, \ldots, N-1
$$

\footnotetext{
Manuscript received September 09, 2008; accepted November 28, 2008. First published January 13, 2009; current version published March 11, 2009. The associate editor coordinating the review of this manuscript and approving it for publication was Dr. Biao Chen. This work was supported in part by the Swedish Research Council, the European Research Council, the Army Research Office under Grant W911NF-07-1-0450, the Office of Naval Research under Grants N00014-09-1-0211 and N00014-07-1-0193, and the National Science Foundation under Grant CCF-0634786.

P. Stoica is with the Department of Information Technology, Uppsala University, Uppsala, Sweden (e-mail: ps@it.uu.se).

$\mathrm{H}$. He and J. Li are with the Department of Electrical and Computer Engineering, University of Florida, Gainesville, FL 32611-6130 USA (e-mail: haohe@ufl.edu; li@dsp.ufl.edu).

Digital Object Identifier 10.1109/TSP.2009.2012562
}

be the correlation function of $\left\{x_{n}\right\}_{n=1}^{N}$, where $(\cdot)^{*}$ denotes the complex conjugate for scalars and the conjugate transpose for vectors and matrices, and let

$$
\mathrm{ISL}=\sum_{k=1}^{N-1}\left|r_{k}\right|^{2}
$$

be the integrated sidelobe level (ISL) metric. The main focus of this paper is on algorithms for minimizing the ISL metric or ISL-related metrics over the set of unimodular sequences. Note that the minimization of the ISL metric is equivalent to the maximization of the merit factor (MF) defined as follows:

$$
\mathrm{MF}=\frac{\left|r_{0}\right|^{2}}{\sum_{\substack{k=-(N-1) \\ k \neq 0}}^{N-1}\left|r_{k}\right|^{2}}=\frac{N^{2}}{2 \mathrm{ISL}} .
$$

Unimodular sequences with large MF values are desired in many applications, including wireless communications and range compression radar and sonar. In these applications, an emitted (probing or training) sequence with a large MF reduces the risk that the received sequence of interest is drawn in correlated multipath or clutter interferences. Additionally, the limitations of the sequence generation hardware (including the analog-to-digital conversion parts) lead to the requirement that the emitted sequence be unimodular.

Owing to the significant theoretical and practical interest in the design of unimodular sequences with good correlation properties (in particular, with large MF values), it should come as no surprise that the literature on this topic is extensive; see [1]-[21] and the many references therein.

Because the ISL metric may be highly multimodal (i.e., it may have multiple local minima), stochastic optimization algorithms have been suggested for its minimization. However, the computational burden of these algorithms becomes prohibitive as $N$ increases: such algorithms are hardly effective on the currently available computing machines for $N \sim 10^{3}$ or larger. Optimization algorithms for locally minimizing the ISL metric have also been proposed. These algorithms can be used to provide quick solutions to the problem of reducing the ISL value of a given reasonably good sequence. They can also be used as local minimization blocks of a stochastic global optimization algorithm. However, most of the existing local minimization algorithms for the ISL metric are descent gradient methods whose convergence problems as well as computational burdens increase significantly as $N$ increases.

In this paper, we introduce several cyclic algorithms (CAs) for the local minimization of ISL-related metrics. The first algorithm is an extension of the CA in [21] (see also [19] and [20]), 
which we call CA-pruned (CAP). CAP deals with a weighted ISL (WISL) metric of the form

$$
\begin{aligned}
\mathrm{WISL} & =\sum_{k=1}^{N-1} w_{k}\left|r_{k}\right|^{2} \\
w_{k} & \geq 0, \quad k=1, \ldots, N-1
\end{aligned}
$$

corresponding to a particular set of weights $\left\{w_{k}\right\}_{k=1}^{N-1}$ (see Section II for details). Such weighted ISL metrics are important in applications where we want to reduce, as much as possible, the interference due to a known multipath or a known clutter discrete. CAP requires the singular value decomposition (SVD) of a matrix of dimension on the order of $N$, so it might be difficult to run on a PC for values of $N$ much larger than $N \sim 10^{3}$. With this problem of CAP in mind, we introduce a new CA called CAN (CA-new) that can be used for the local minimization of the unweighted ISL metric [i.e., (5) with $w_{k} \equiv 1$ ]. CAN is based on fast Fourier transform (FFT) operations and can be used virtually for any practically relevant values of $N$ up to $N \sim 10^{6}$ or even larger. We also modify CAN so that it can tackle arbitrary weights, i.e., $\left\{w_{k}\right\}_{k=1}^{N-1}$ in (5) can be chosen as any nonnegative real numbers. The resulting algorithm, which is called WeCAN (weighted CAN), requires $N$ times more computations than CAN and can be run on a PC for $N$ up to $N \sim 10^{4}$.

\section{CAP}

Let

$$
\mathbf{X}=\left[\begin{array}{ccc}
x_{1} & & 0 \\
\vdots & \ddots & \\
x_{N} & & x_{1} \\
& \ddots & \vdots \\
0 & & x_{N}
\end{array}\right]_{(2 N-1) \times N}
$$

and observe that

$$
\mathbf{X}^{*} \mathbf{X}=\left[\begin{array}{cccc}
r_{0} & r_{1}^{*} & \cdots & r_{N-1}^{*} \\
r_{1} & r_{0} & \ddots & \vdots \\
\vdots & \ddots & \ddots & r_{1}^{*} \\
r_{N-1} & \cdots & r_{1} & r_{0}
\end{array}\right]_{N \times N}
$$

Because $\mathbf{X}^{*} \mathbf{X} \approx N \mathbf{I}$ for a sequence with good autocorrelation properties, we can think of designing $\left\{x_{n}\right\}_{n=1}^{N}$ by minimizing the following criterion:

$$
\left\|\mathbf{X}^{*} \mathbf{X}-N \mathbf{I}\right\|^{2}
$$

over the set of unimodular sequences; hereafter, $\|\cdot\|$ denotes the Frobenius matrix norm. However, the above criterion is a quartic function of $\left\{x_{n}\right\}$ that is relatively difficult to tackle. With this fact in mind, [21] (see also [19] and [20]) has suggested replacing (8) with the following simpler criterion (which is a quadratic function of the unknowns):

$$
\|\mathbf{X}-\sqrt{N} \mathbf{Q}\|^{2}
$$

where $\mathbf{Q}$ is a $(2 N-1) \times N$ semiunitary matrix (i.e., $\mathbf{Q}^{*} \mathbf{Q}=\mathbf{I}$ ). The design problem associated with (9) can be stated as follows:

$$
\begin{aligned}
& \min _{\left\{x_{n}\right\}_{n=1}^{N} ; \mathbf{Q}}\|\mathbf{X}-\sqrt{N} \mathbf{Q}\|^{2} \\
& \text { subject to } \quad \mathbf{Q}^{*} \mathbf{Q}=\mathbf{I} \\
& \quad\left|x_{n}\right|=1, \quad n=1, \ldots, N .
\end{aligned}
$$

Note that the problems of minimizing (8) and, respectively, (9) are not equivalent (these two problems may well have different solutions $\left\{x_{n}\right\}$ ), yet they are "almost equivalent" in the sense that if the criterion in (9) takes on a small value, then so does (8), and vice versa. More specifically, it is clear that (8) is equal to zero if and only if (9) is equal to zero. Consequently, by continuity arguments, if the global minimum value of (8) is "sufficiently small," then the sequences minimizing (8) and, respectively, (9) can be expected to be close to one another. Put differently, in such a case the sequence minimizing (8) leads to a small value of (9), and vice versa. However, as already mentioned, the two sequences that minimize (8) and, respectively, (9) will in general be different from one another. Furthermore, the local minima of the two criteria will in general be different; in particular, they can occur at sequences that are not the same for (8) and for (9). A more quantitative mathematical analysis of the global and local minima of the two criteria, as well as of the way in which they relate to each other, appears to be a difficult task that falls beyond the scope of this paper.

Remark: As already pointed out in Section I, the case of finite-alphabet sequences can also be dealt with in our framework. However, the performance of the resulting algorithms might not be as satisfactory as that corresponding to the general unimodular case. One possible explanation of this fact is related to the above discussion on (8) and (9): when we add more constraints on $\left\{x_{n}\right\}$, such as a finite alphabet requirement, the minimum value of the criterion in (8) may increase quite a bit, and therefore the "almost equivalence" between (8) and (9) may cease to hold true. Another explanation might be that the number of local minima of the ISL (or WISL) metric tends to increase as more constraints are imposed on $\left\{x_{n}\right\}$ (with the binary case being the most constrained one). Consequently, it becomes more difficult to find a sequence $\left\{x_{n}\right\}$ such that the criterion in (8) or (9) takes on a small value when a finite-alphabet constraint is enforced.

In contrast to (8), the derivation of a CA for (10) is relatively straightforward ([19]-[21]). However, this is not to say that the derivation of a CA for the criterion in (8) is infeasible. In fact, such a CA can be derived, as we show in the forthcoming paper [22] that deals with vector sequence design. The problem with such an algorithm for (8) is that, due to the more complicated form of the criterion, it is often much slower than a CA for the "almost equivalent" criterion in (10).

We will not discuss explicitly a CA for (10) because it considers all correlation lags $\left\{r_{k}\right\}_{k=0}^{N-1}$, which is somewhat infrequently required in applications and therefore results in an unnecessary increase of the computational burden. Indeed, in many cases, the maximum difference between the arrival times of the sequence of interest and of the interferences is 
(much) smaller than the duration of the emitted sequence (see, e.g., [16], [17], [21], and [23]). Consequently, in such cases, the interest lies in making $\left\{\left|r_{k}\right|\right\}_{k=1}^{P-1}$ small, for some $P<N$, instead of trying to make all correlation sidelobes $\left\{\left|r_{k}\right|\right\}_{k=1}^{N-1}$ small; here the value of $P$ is selected based on a priori knowledge about the application at hand (for instance, in wireless communications, it is usually known that significant channel tap coefficients can occur up to a certain maximum delay, and so we can choose $P$ as the said delay). More generally, we may have a priori information that not even all $\left\{\left|r_{k}\right|\right\}_{k=1}^{P-1}$, but only some of them, need to be made small. In such a case, instead of considering the "all-lag" $\mathbf{X}$ in (6), we consider the following "pruned" matrix:

$$
\tilde{\mathbf{X}}=\overline{\mathbf{X}} \mathbf{T}
$$

of dimension $(N+P-1) \times Q$, where $Q \leq P \leq N$ and $\overline{\mathbf{X}}$ is a truncated version of the $\mathbf{X}$ in (6)

$$
\overline{\mathbf{X}}=\left[\begin{array}{ccc}
x_{1} & & 0 \\
\vdots & \ddots & \\
\vdots & & x_{1} \\
x_{N} & & \vdots \\
& \ddots & \vdots \\
0 & & x_{N}
\end{array}\right]_{(N+P-1) \times P}
$$

and where the $P \times Q$ matrix $\mathbf{T}$ is made from $Q$ selected columns of the $P \times P$ identity matrix, for example

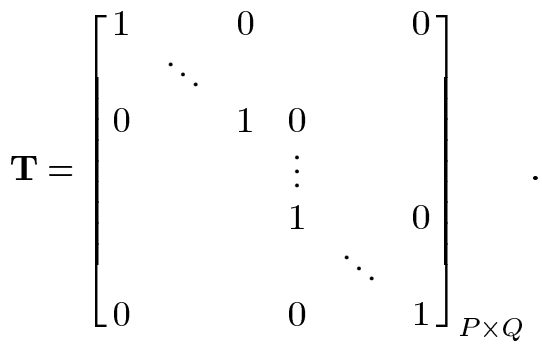

The above $Q$ columns correspond to the $Q$ correlations of interest chosen from $r_{0}, r_{1}, \ldots, r_{P-1}$. With the above notation, the design problem of interest is obtained by modifying (10) as follows:

$$
\begin{aligned}
\min _{\left\{x_{n}\right\}_{n=1}^{N} ; \mathbf{U}} & \|\tilde{\mathbf{X}}-\sqrt{N} \mathbf{U}\|^{2} \\
\text { s.t. } & \mathbf{U}^{*} \mathbf{U}=\mathbf{I} \\
& \left|x_{n}\right|=1, \quad n=1, \ldots, N
\end{aligned}
$$

where $\mathbf{U}$ is an $(N+P-1) \times Q$ semiunitary matrix.

Remark: Equation (14) is "almost equivalent" to minimizing $\left\|\tilde{\mathbf{X}}^{*} \tilde{\mathbf{X}}-N \mathbf{I}\right\|^{2}$. When $Q=P$, we have

$$
\tilde{\mathbf{X}}^{*} \tilde{\mathbf{X}}=\left[\begin{array}{cccc}
r_{0} & r_{1}^{*} & \cdots & r_{P-1}^{*} \\
r_{1} & r_{0} & \ddots & \vdots \\
\vdots & \ddots & \ddots & r_{1}^{*} \\
r_{P-1} & \cdots & r_{1} & r_{0}
\end{array}\right]_{P \times P}
$$

which shows that in this case, CAP implicitly assumes the weight of $w_{k}=2(P-k)$ for $r_{k}(k=1, \ldots, P-1)$ in the
WISL metric in (5), and zero weights for the other correlation lags. When $Q<P, \tilde{\mathbf{X}}^{*} \tilde{\mathbf{X}}$ is no longer a Toeplitz matrix and a general expression for $w_{k}$ does not exist anymore. Roughly speaking, the number of times that $r_{k}$ (together with $r_{k}^{*}$ ) appears in the matrix $\tilde{\mathbf{X}}^{*} \tilde{\mathbf{X}}$ determines the corresponding weight $w_{k}$.

Regarding the minimization problem in (14), we note the following facts. For given $\tilde{\mathbf{X}}$, let

$$
\tilde{\mathbf{X}}^{*}=\mathbf{U}_{1} \boldsymbol{\Sigma} \mathbf{U}_{2}^{*}
$$

denote the SVD of $\tilde{\mathbf{X}}^{*}$ (here $\mathbf{U}_{1}$ is a $Q \times Q$ unitary matrix, $\mathbf{U}_{2}$ is an $(N+P-1) \times Q$ semiunitary matrix, and $\Sigma$ is a $Q \times Q$ diagonal matrix). Then the solution $\mathbf{U}$ of (14), for fixed $\tilde{\mathbf{X}}$, is given by (see [19] or the references there and in [20])

$$
\mathbf{U}=\mathbf{U}_{2} \mathbf{U}_{1}^{*}
$$

Next note that, for given $\mathbf{U}$, the minimization of (14) with respect to $\left\{x_{n}\right\}_{n=1}^{N}$ also has a simple closed-form solution. To see this, let $x$ denote an arbitrary element of the sequence $\left\{x_{n}\right\}_{n=1}^{N}$. Then it follows from (14) that the generic form of the minimization problem with respect to the elements of $\left\{x_{n}\right\}_{n=1}^{N}$ is

$$
\min _{x} \sum_{k=1}^{Q}\left|x-\mu_{k}\right|^{2}
$$

where $\left\{\mu_{k}\right\}_{k=1}^{Q}$ are the elements of the matrix $\sqrt{N} \mathbf{U}$ whose positions are the same as the positions of $x$ in $\tilde{\mathbf{X}}$. (As an example, let us assume that $Q=P$ and therefore that $\tilde{\mathbf{X}}=\overline{\mathbf{X}}$. Then, for $x=x_{n}$, the corresponding sequence $\left\{\mu_{k}\right\}_{k=1}^{P}$ is given by the $(n-1+i, i)$ th elements of $\sqrt{N} \mathbf{U}$, for $i=1, \ldots, P$.) Because $|x|=1$, the criterion in (18) can be rewritten as

$$
\begin{aligned}
\sum_{k=1}^{Q}\left|x-\mu_{k}\right|^{2}= & \text { const }-2 \operatorname{Re}\left[x \sum_{k=1}^{Q} \mu_{k}^{*}\right] \\
= & \text { const }-2\left|\sum_{k=1}^{Q} \mu_{k}\right| \\
& \cdot \cos \left[\arg (x)-\arg \left(\sum_{k=1}^{Q} \mu_{k}\right)\right] .
\end{aligned}
$$

Hence the minimizer $x$ of the criterion in (18) is given by

$$
x=e^{j \phi}, \quad \phi=\arg \left(\sum_{k=1}^{Q} \mu_{k}\right) .
$$

The CAP for the cyclic minimization of the criterion in (14) follows from the above discussion as a natural corollary.

CAP

- Step 0) Set the matrix $\tilde{\mathbf{X}}$ to an initial value (e.g., $\left\{x_{n}\right\}_{n=1}^{N}$ can be set to $\left\{e^{j 2 \pi \theta_{n}}\right\}_{n=1}^{N}$, where $\left\{\theta_{n}\right\}_{n=1}^{N}$ are independent random variables uniformly distributed in $[0,2 \pi]$ or $\left\{x_{n}\right\}_{n=1}^{N}$ can be initialized by a good existing sequence such as a Golomb sequence [12]).

- Step 1) Compute the semiunitary matrix $\mathbf{U}$ that minimizes (14) for $\left\{x_{n}\right\}_{n=1}^{N}$ fixed at its most recent value [see (16) and (17)]. 
- Step 2) Compute the sequence $\left\{x_{n}\right\}_{n=1}^{N}$ that minimizes (14), under the constraint $\left|x_{n}\right|=1$, for $\mathbf{U}$ fixed at its most recent value [see (20)].

- Iteration: repeat Steps 1) and 2) until some stop criterion is satisfied, e.g., $\left\|\mathbf{x}^{(i)}-\mathbf{x}^{(i+1)}\right\|<\epsilon$, where $\mathbf{x}^{(i)}$ is the sequence obtained at the $i$ th iteration and $\epsilon$ is a predefined threshold (see the remark in Section V-C for a brief discussion about how to choose the value of $\epsilon$ ).

The SVD of the $Q \times(N+P-1)$ matrix $\tilde{\mathrm{X}}^{*}$ in (16) is relatively computationally intensive for large values of $N$ and $Q$. As a rough rule of thumb, on a regular PC, the use of CAP may be limited to values of $N \sim 10^{3}$ depending on how many correlation lags are considered. In the next section, we introduce a new CA (CAN = CA-new) for the local minimization of the unweighted ISL metric that does not have such a limitation: indeed, CAN can be used with values of $N \sim 10^{6}$ or even larger if so desired.

\section{CAN}

The derivation of CAN involves several steps, the first of which consists of expressing the ISL metric in the frequency domain. It is well known that, for any $\omega \in[0,2 \pi]$

$$
\left|\sum_{n=1}^{N} x_{n} e^{-j \omega n}\right|^{2}=\sum_{k=-(N-1)}^{N-1} r_{k} e^{-j \omega k} \triangleq \Phi(\omega)
$$

(see, e.g., [24]). Furthermore, it can be shown that the ISL metric in (3) can be equivalently written as

$$
\mathrm{ISL}=\frac{1}{4 N} \sum_{p=1}^{2 N}\left[\Phi\left(\omega_{p}\right)-N\right]^{2}
$$

where $\left\{\omega_{p}\right\}$ are the following Fourier frequencies:

$$
\omega_{p}=\frac{2 \pi}{2 N} p, \quad p=1, \ldots, 2 N .
$$

[Note that (22) is a Parseval-type equality.] To prove (22), let $\delta_{k}$ denote the Kronecker delta

$$
\delta_{k}= \begin{cases}1, & \text { for } k=0 \\ 0, & \text { for } k \neq 0\end{cases}
$$

and use the correlogram-based expression for $\Phi(\omega)$ in (21) to verify that

$$
\begin{aligned}
\sum_{p=1}^{2 N} & {\left[\Phi\left(\omega_{p}\right)-N\right]^{2} } \\
= & \sum_{p=1}^{2 N}\left[\sum_{k=-(N-1)}^{N-1}\left(r_{k}-N \delta_{k}\right) e^{-j \omega_{p} k}\right]^{2} \\
= & \sum_{k=-(N-1)}^{N-1} \sum_{\tilde{k}=-(N-1)}^{N-1}\left(r_{k}-N \delta_{k}\right)\left(r_{\tilde{k}}-N \delta_{\tilde{k}}\right)^{*} \\
& \times\left[\sum_{p=1}^{2 N} e^{-j \omega_{p}(k-\tilde{k})}\right] .
\end{aligned}
$$

Because, for $|k-\tilde{k}| \leq 2 N-2$

$$
\begin{aligned}
\sum_{p=1}^{2 N} e^{-j \omega_{p}(k-\tilde{k})} & =e^{-j \frac{2 \pi}{2 N}(k-\tilde{k})} \cdot \frac{e^{-j 2 \pi(k-\tilde{k})}-1}{e^{-j \frac{2 \pi}{2 N}(k-\tilde{k})}-1} \\
& =2 N \delta_{(k-\tilde{k})}
\end{aligned}
$$

we obtain from (24) the following equation:

$$
\begin{aligned}
\frac{1}{4 N} \sum_{p=1}^{2 N}\left[\Phi\left(\omega_{p}\right)-N\right]^{2} & =\frac{1}{2} \sum_{k=-(N-1)}^{N-1}\left|r_{k}-N \delta_{k}\right|^{2} \\
& =\sum_{k=1}^{N-1}\left|r_{k}\right|^{2}=\text { ISL }
\end{aligned}
$$

which is (22). Using the periodogram-based expression for $\Phi(\omega)$ [see (21)] in (22) shows that the problem of minimizing the ISL is equivalent to the minimization of the following frequency-domain metric:

$$
\sum_{p=1}^{2 N}\left[\left|\sum_{n=1}^{N} x_{n} e^{-j \omega_{p} n}\right|^{2}-N\right]^{2}
$$

This equivalence result has an obvious intuitive interpretation: minimizing the ISL makes the sequence behave like white noise, and consequently its periodogram should be nearly constant in frequency.

The next point to note is that the criterion in (27) is a quartic function of $\left\{x_{n}\right\}$. However, using the same type of argument as the one that led from (8) to (9), we can readily verify that the minimization of (27) with respect to $\left\{x_{n}\right\}$ is "almost equivalent" to the following simpler problem (whose criterion is a quadratic function of $\left\{x_{n}\right\}$ ):

$$
\min _{\left\{x_{n}\right\}_{n=1}^{N} ;\left\{\psi_{p}\right\}_{p=1}^{2 N}} \sum_{p=1}^{2 N}\left|\sum_{n=1}^{N} x_{n} e^{-j \omega_{p} n}-\sqrt{N} e^{j \psi_{p}}\right|^{2} .
$$

Let

$$
\mathbf{a}_{p}^{*}=\left[\begin{array}{lll}
e^{-j \omega_{p}} & \cdots & e^{-j 2 N \omega_{p}}
\end{array}\right]
$$

let $\mathbf{A}^{*}$ be the following unitary $2 N \times 2 N$ FFT matrix:

$$
\mathbf{A}^{*}=\frac{1}{\sqrt{2 N}}\left[\begin{array}{c}
\mathbf{a}_{1}^{*} \\
\vdots \\
\mathbf{a}_{2 N}^{*}
\end{array}\right]
$$

and let $\mathbf{z}$ be the sequence $\left\{x_{n}\right\}_{n=1}^{N}$ padded with $N$ zeros

$$
\mathbf{z}=\left[\begin{array}{llllll}
x_{1} & \cdots & x_{N} & 0 & \cdots & 0
\end{array}\right]_{2 N \times 1}^{T} .
$$

Then the criterion in (28) can be rewritten in the following more compact form (to within a multiplicative constant):

$$
\left\|\mathbf{A}^{*} \mathbf{z}-\mathbf{v}\right\|^{2}
$$

where

$$
\mathbf{v}=\frac{1}{\sqrt{2}}\left[\begin{array}{lll}
e^{j \psi_{1}} & \cdots & e^{j \psi_{2 N}}
\end{array}\right]^{T} .
$$


For given $\left\{x_{n}\right\}$, the minimization of (32) with respect to $\left\{\psi_{p}\right\}$ is immediate: let

$$
\mathbf{f}=\mathbf{A}^{*} \mathbf{z}
$$

denote the FFT of $\mathbf{z}$; then

$$
\psi_{p}=\arg \left(f_{p}\right), \quad p=1, \ldots, 2 N .
$$

Similarly, for given $\mathbf{v}$, let

$$
\mathrm{g}=\mathbf{A v}
$$

denote the inverse FFT of $\mathbf{v}$. Because $\left\|\mathbf{A}^{*} \mathbf{z}-\mathbf{v}\right\|^{2}=\|\mathbf{z}-\mathbf{A v}\|^{2}$, it follows that the minimizing sequence $\left\{x_{n}\right\}$ is given by

$$
x_{n}=e^{j \arg \left(g_{n}\right)}, \quad n=1, \ldots, N .
$$

The CAN for the cyclic local minimization of the ISL-related metric in (28) can now be summarized as follows.

\section{CAN}

- Step 0) Set the $\left\{x_{n}\right\}_{n=1}^{N}$ to some initial values (e.g., $\left\{x_{n}\right\}_{n=1}^{N}$ can be randomly generated or given by a good existing sequence, as mentioned in the CAP algorithm in Section II).

- Step 1) Compute the $\left\{\psi_{p}\right\}_{p=1}^{2 N}$ that minimize the metric for $\left\{x_{n}\right\}_{n=1}^{N}$ fixed at their most recent values [see (35)].

- Step 2) Compute the sequence $\left\{x_{n}\right\}_{n=1}^{N}$ that minimizes the metric, under the constraint $\left|x_{n}\right|=1$, for $\left\{\psi_{p}\right\}_{p=1}^{2 N}$ fixed at their most recent values [see (37)].

- Iteration: repeat Steps 1) and 2) until a prespecified stop criterion is satisfied, e.g., $\left\|\mathbf{x}^{(i)}-\mathbf{x}^{(i+1)}\right\|<\epsilon$, where $\mathbf{x}^{(i)}$ is the sequence obtained at the $i$ th iteration and $\epsilon$ is a predefined threshold, such as $10^{-3}$.

Owing to its simple (I)FFT operations, CAN can be used for very large values of $N$, such as $N \sim 10^{6}$.

In the next section, we present an extended version of CAN that can deal with the WISL metric (with arbitrarily chosen weights) as defined in (5). The extended algorithm is called WeCAN (weighted CAN). The price paid for WeCAN's ability to deal with a general WISL metric is an increased computational burden compared to CAN. Specifically, as will be shown in the next section, each iteration of WeCAN requires $N$ computations of $2 N$-point (I)FFTs; thus the number of flops required by WeCAN is roughly $N$ times larger than that of CAN. Nonetheless, WeCAN can still be used for relatively large values of $N$, such as $N \sim 10^{4}$.

\section{WECAN}

Similarly to the proof of (22) in Section III, we can derive the following expression for the WISL metric $\left(\gamma_{k}\right.$ below is related to the weight $w_{k}$ in (5) as $w_{k}=\gamma_{k}^{2}$ ):

$$
\begin{aligned}
\mathrm{WISL} & =\sum_{k=1}^{N-1} \gamma_{k}^{2}\left|r_{k}\right|^{2} \\
& =\frac{1}{4 N} \sum_{p=1}^{2 N}\left[\tilde{\Phi}\left(\omega_{p}\right)-\gamma_{0} N\right]^{2}
\end{aligned}
$$

where

$$
\begin{aligned}
\tilde{\Phi}\left(\omega_{p}\right) & \triangleq \sum_{k=-(N-1)}^{N-1} \gamma_{k} r_{k} e^{-j \omega_{p} k} \\
\omega_{p} & =\frac{2 \pi}{2 N} p, \quad p=1, \ldots, 2 N
\end{aligned}
$$

and where $\left\{\gamma_{k}\right\}_{k=1}^{N-1}$ are real-valued (with $\gamma_{k}=\gamma_{-k}$ ). Note that by choosing $\left\{\gamma_{k}\right\}_{k=1}^{N-1}$ appropriately, we can weigh the correlation lags in (38) in any desired way. Regarding $\gamma_{0}$, which does not enter into (38), it will be chosen to ensure that the matrix

$$
\boldsymbol{\Gamma}=\frac{1}{\gamma_{0}}\left[\begin{array}{cccc}
\gamma_{0} & \gamma_{1} & \cdots & \gamma_{N-1} \\
\gamma_{1} & \gamma_{0} & \ddots & \vdots \\
\vdots & \ddots & \ddots & \gamma_{1} \\
\gamma_{N-1} & \cdots & \gamma_{1} & \gamma_{0}
\end{array}\right]
$$

is positive semidefinite, which we denote by $\Gamma \geq 0$. This can be done in the following simple way: let $\tilde{\boldsymbol{\Gamma}}$ be the matrix $\gamma_{0} \boldsymbol{\Gamma}$ with all diagonal elements set to zero and let $\lambda_{\min }$ denote the minimum eigenvalue of $\tilde{\Gamma}$; then $\Gamma \geq 0$ if and only if $\gamma_{0}+\lambda_{\min } \geq$ 0 , a condition that can always be satisfied by selecting $\gamma_{0}$.

Next we will derive a criterion that is "almost equivalent" to (39) and that depends quadratically on the unknowns $\left\{x_{n}\right\}_{n=1}^{N}$, similarly to what we have done in the previous sections. To do so, we must apparently obtain a square root of $\tilde{\Phi}\left(\omega_{p}\right)$ in (40) that is linear in $\left\{x_{n}\right\}_{n=1}^{N}$. Note the following discrete Fourier transform pairs:

$$
\begin{aligned}
\left\{r_{k}\right\} & \longleftrightarrow \Phi(\omega)=|X(\omega)|^{2} \\
\left\{\gamma_{k} r_{k}\right\} & \longleftrightarrow \tilde{\Phi}(\omega)=\Gamma(\omega) *|X(\omega)|^{2}
\end{aligned}
$$

where

$$
X(\omega)=\sum_{n=1}^{N} x_{n} e^{-j n \omega}, \quad \Gamma(\omega)=\sum_{k=-(N-1)}^{N-1} \gamma_{k} e^{-j \omega k}
$$

and where $*$ is the convolution operator. Thus $\tilde{\Phi}\left(\omega_{p}\right)$ can be expressed as

$$
\begin{aligned}
\tilde{\Phi}\left(\omega_{p}\right)= & \frac{1}{2 \pi} \int_{-\pi}^{\pi} \Gamma\left(\omega_{p}-\psi\right)|X(\psi)|^{2} d \psi \\
= & \frac{1}{2 \pi} \int_{-\pi}^{\pi} \sum_{k=-(N-1)}^{N-1} \gamma_{k} e^{-j k\left(\omega_{p}-\psi\right)} \sum_{n=1}^{N} x_{n} e^{-j n \psi} \\
& \times \sum_{\tilde{n}=1}^{N} x_{\tilde{n}}^{*} e^{j \tilde{n} \psi} d \psi \\
= & \sum_{k=-(N-1)}^{N-1} \sum_{n=1}^{N} \sum_{\tilde{n}=1}^{N} \gamma_{k} x_{n} x_{\tilde{n}}^{*} \\
& \times\left\{\frac{1}{2 \pi} \int_{-\pi}^{\pi} e^{j \psi(k-n+\tilde{n})} d \psi\right\} e^{-j \omega_{p} k}
\end{aligned}
$$


It is easy to verify that

$$
\frac{1}{2 \pi} \int_{-\pi}^{\pi} e^{j \psi(k-n+\tilde{n})} d \psi=\delta_{k-(n-\tilde{n})} .
$$

Thus

$$
\begin{aligned}
\tilde{\Phi}\left(\omega_{p}\right) & =\sum_{n=1}^{N} \sum_{\tilde{n}=1}^{N} \gamma_{n-\tilde{n}} x_{n} x_{\tilde{n}}^{*} e^{-j \omega_{p}(n-\tilde{n})} \\
& =\tilde{\mathbf{x}}_{p}^{*}\left(\gamma_{0} \boldsymbol{\Gamma}\right) \tilde{\mathbf{x}}_{p}
\end{aligned}
$$

where

$$
\tilde{\mathbf{x}}_{p}=\left[\begin{array}{llll}
x_{1} e^{-j \omega_{p}} & x_{2} e^{-j 2 \omega_{p}} & \cdots & x_{N} e^{-j N \omega_{p}}
\end{array}\right]^{T}
$$

and $\boldsymbol{\Gamma}$ is defined in (41). Therefore, the WISL metric in (39) can be written as

$$
\mathrm{WISL}=\frac{\gamma_{0}^{2}}{4 N} \sum_{p=1}^{2 N}\left[\tilde{\mathbf{x}}_{p}^{*} \boldsymbol{\Gamma} \tilde{\mathbf{x}}_{p}-N\right]^{2} .
$$

This expression suggests that the following problem can be expected to be "almost equivalent" to the minimization of the WISL metric:

$$
\begin{aligned}
& \min _{\left\{x_{n}\right\}_{n=1}^{N},\left\{\boldsymbol{\alpha}_{p}\right\}_{p=1}^{2 N}} \sum_{p=1}^{2 N}\left\|\mathbf{C} \tilde{\mathbf{x}}_{p}-\boldsymbol{\alpha}_{p}\right\|^{2} \\
& \text { s.t. } \quad\left\|\boldsymbol{\alpha}_{p}\right\|^{2}=N, \quad p=1, \ldots, 2 N, \\
&\left|x_{n}\right|=1, \quad n=1, \ldots, N
\end{aligned}
$$

where the $N \times N$ matrix $\mathbf{C}$ is a square root of $\boldsymbol{\Gamma}$, i.e., $\Gamma=\mathbf{C}^{T} \mathbf{C}$.

A cyclic algorithm for (49), which we will call WeCAN, can be derived as follows. For given $\left\{x_{n}\right\}_{n=1}^{N}$, (49) decouples into $2 N$ independent problems, each of which has the following form:

$$
\begin{array}{ll}
\min _{\boldsymbol{\alpha}_{p}} & \left\|\mathbf{f}_{p}-\boldsymbol{\alpha}_{p}\right\|^{2} \\
\text { s.t. } & \left\|\boldsymbol{\alpha}_{p}\right\|^{2}=N
\end{array}
$$

where the $N \times 1$ vector $\mathbf{f}_{p}=\mathbf{C} \tilde{\mathbf{x}}_{p}$ is given. Note that under the constraint $\left\|\boldsymbol{\alpha}_{p}\right\|^{2}=N$, we have

$$
\begin{aligned}
\left\|\mathbf{f}_{p}-\boldsymbol{\alpha}_{p}\right\|^{2} & =\text { const }-2 \operatorname{Re}\left\{\mathbf{f}_{p}^{*} \boldsymbol{\alpha}_{p}\right\} \\
& \geq \text { const }-2\left\|\mathbf{f}_{p}\right\|\left\|\boldsymbol{\alpha}_{p}\right\|=\text { const }-2 N\left\|\mathbf{f}_{p}\right\|
\end{aligned}
$$

where the equality is achieved if and only if

$$
\boldsymbol{\alpha}_{p}=\sqrt{N} \frac{\mathbf{f}_{p}}{\left\|\mathbf{f}_{p}\right\|} .
$$

This is therefore the solution to the minimization problem in (49) for given $\left\{x_{n}\right\}_{n=1}^{N}$. Note that the computation of $\left\{\mathbf{f}_{p}\right\}_{p=1}^{2 N}$ can be done by means of the FFT. Indeed, let $c_{k n}$ denote the $(k, n)$ th element of $\mathbf{C}$ and define

$$
\mathbf{z}_{k}=\left[\begin{array}{llllll}
c_{k 1} x_{1} & \cdots & c_{k N} x_{N} & 0 & \cdots & 0
\end{array}\right]_{(2 N \times 1)}^{T}
$$

and

$$
\mathbf{F}=\sqrt{2 N} \mathbf{A}^{*} \cdot\left[\begin{array}{lll}
\mathbf{z}_{1} & \mathbf{z}_{2} & \cdots \\
\mathbf{z}_{N}
\end{array}\right]_{2 N \times N}
$$

where the unitary $2 N \times 2 N$ FFT matrix $\mathbf{A}^{*}$ has been defined in (30). Then it is not difficult to see that the transpose of the vector $\mathbf{f}_{p}$ is given by the $p$ th row of $\mathbf{F}$.

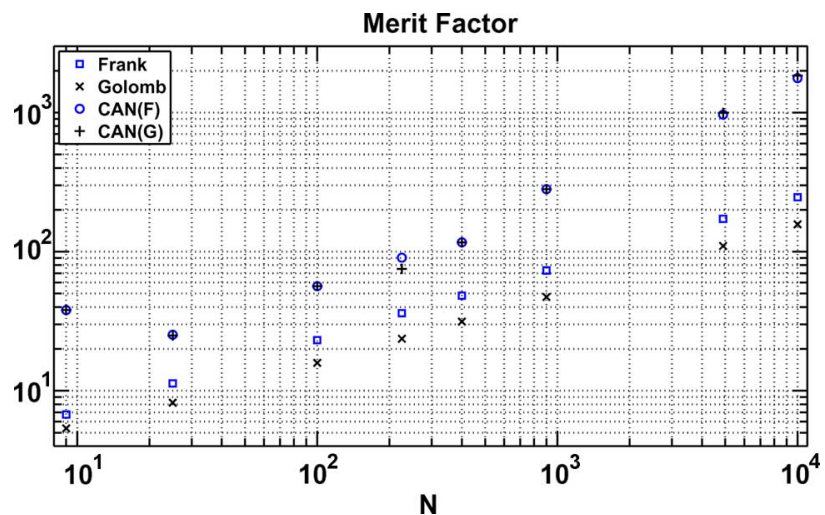

Fig. 1. The merit factors of the Golomb, Frank, CAN(G), and CAN(F) sequences of lengths from $3^{2}$ up to $100^{2}$.

Next we show that, for given $\left\{\boldsymbol{\alpha}_{p}\right\}_{p=1}^{2 N}$, the minimization problem in (49) with respect to $\left\{x_{n}\right\}_{n=1}^{N}$ also has a closed-form solution. Let $\alpha_{p k}$ denote the $k$ th element of $\boldsymbol{\alpha}_{p}$ and let $\mathbf{a}_{p}^{*}$ be given by (29). Using this notation, the criterion in (49) can be written as

$$
\begin{aligned}
\sum_{p=1}^{2 N}\left\|\mathbf{C} \tilde{\mathbf{x}}_{p}-\boldsymbol{\alpha}_{p}\right\|^{2} & =\sum_{k=1}^{N} \sum_{p=1}^{2 N}\left|\mathbf{a}_{p}^{*} \mathbf{z}_{k}-\alpha_{p k}\right|^{2} \\
& =\sum_{k=1}^{N}\left\|\mathbf{A}^{*} \mathbf{z}_{k}-\boldsymbol{\beta}_{k}\right\|^{2} \\
& =\sum_{k=1}^{N}\left\|\mathbf{z}_{k}-\mathbf{A} \boldsymbol{\beta}_{k}\right\|^{2}
\end{aligned}
$$

where

$$
\boldsymbol{\beta}_{k}=\frac{1}{\sqrt{2 N}}\left[\begin{array}{lll}
\alpha_{1 k} & \cdots & \alpha_{2 N, k}
\end{array}\right]^{T}, \quad k=1, \ldots, N .
$$

For a generic element of $\left\{x_{n}\right\}_{n=1}^{N}$, denoted as $x$, (55) becomes

$$
\sum_{k=1}^{N}\left|\mu_{k} x-\nu_{k}\right|^{2}=\text { const }-2 \operatorname{Re}\left[\left(\sum_{k=1}^{N} \mu_{k}^{*} \nu_{k}\right) x^{*}\right]
$$

where $\mu_{k}$ and $\nu_{k}$ are given by the corresponding elements in $\mathbf{z}_{k}$ and $\mathbf{A} \boldsymbol{\beta}_{k}$, respectively. Under the unimodular constraint, the minimizer $x$ of the criterion in (57) is given by

$$
x=e^{j \phi}, \quad \phi=\arg \left(\sum_{k=1}^{N} \mu_{k}^{*} \nu_{k}\right) .
$$

This observation concludes the derivation of the main steps of the WeCAN algorithm, whose summary is as follows.

\section{WeCAN}

- Step 0) Set the $\left\{x_{n}\right\}_{n=1}^{N}$ to some initial values and select the desired weights $\left\{\gamma_{k}\right\}_{k=1}^{N-1}$; also choose $\gamma_{0}$ such that the matrix $\Gamma$ in (41) is positive semidefinite.

- Step 1) Compute the $\left\{\boldsymbol{\alpha}_{p}\right\}_{p=1}^{2 N}$ that minimize the criterion in (49) for $\left\{x_{n}\right\}_{n=1}^{N}$ fixed at their most recent values [see (52)]. 


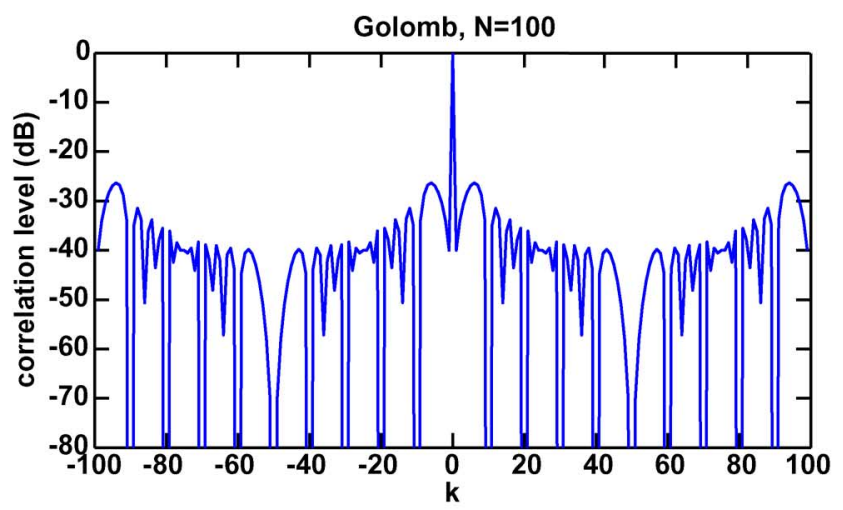

(a)

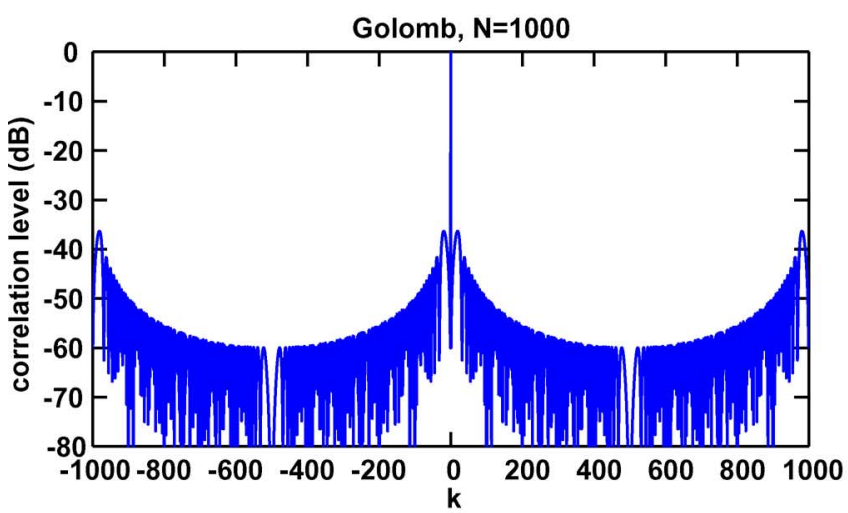

(c)

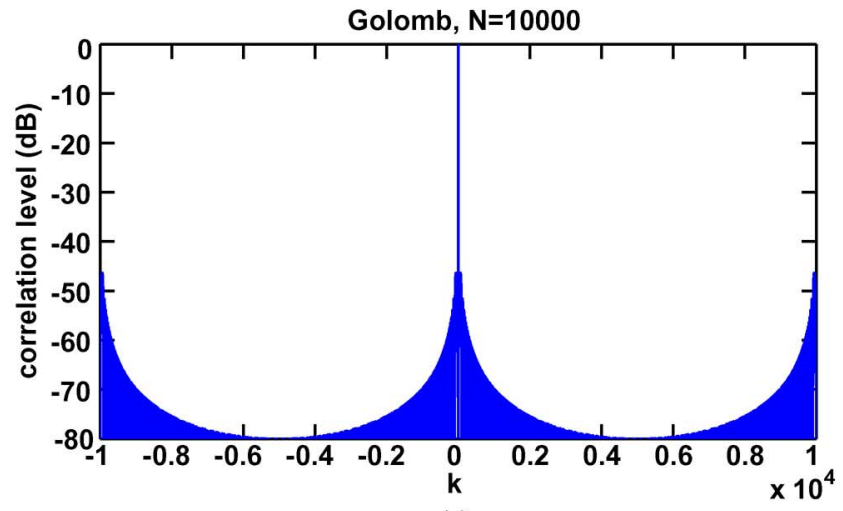

(e)

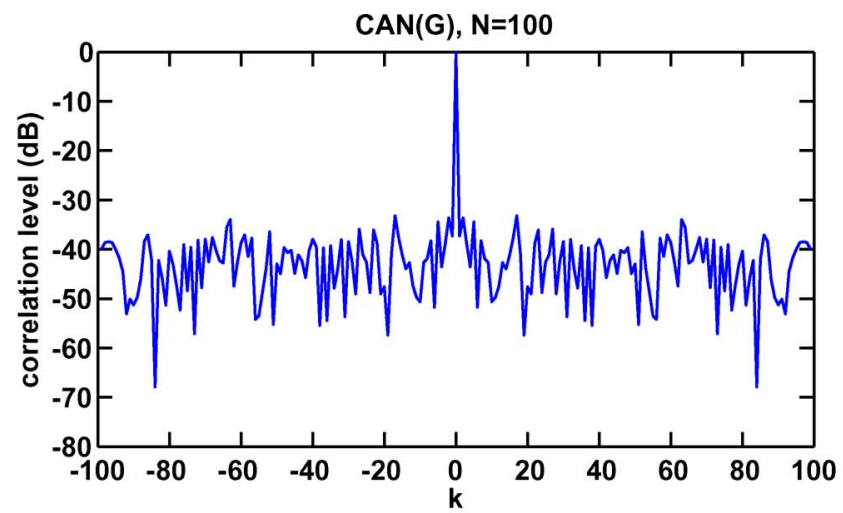

(b)

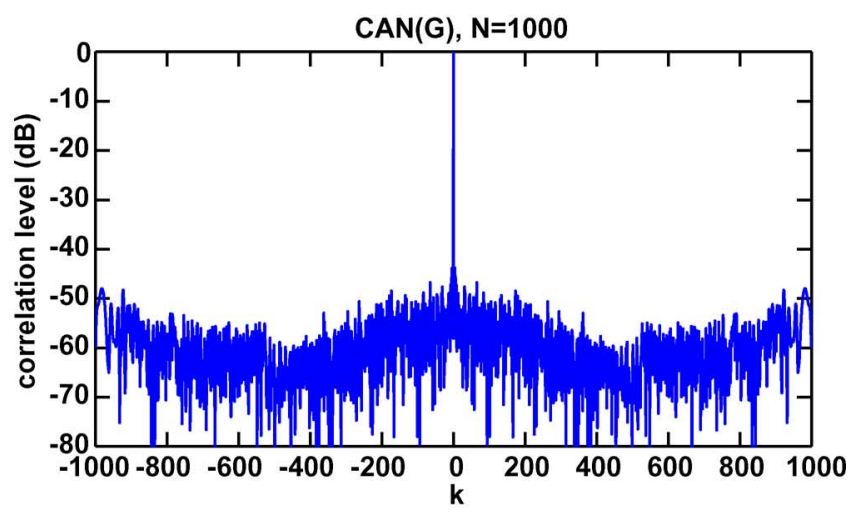

(d)

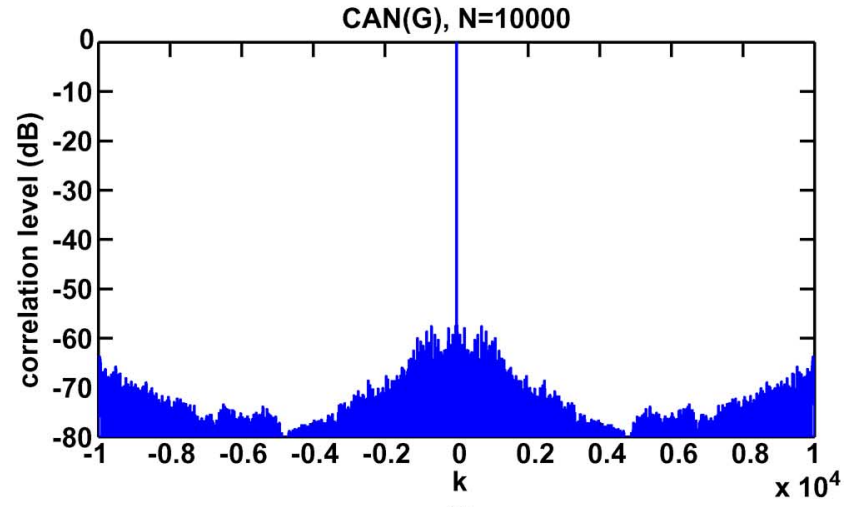

(f)

Fig. 2. Correlation levels of the Golomb and CAN sequences of lengths $N=10^{2}, 10^{3}$, and $10^{4}$ designed under the ISL metric. (a) The Golomb sequence $N=10^{2}$, (b) the $\operatorname{CAN}(\mathrm{G})$ sequence $N=10^{2}$, (c) the Golomb sequence $N=10^{3}$, (d) the $\operatorname{CAN}(\mathrm{G})$ sequence $N=10^{3}$, (e) the Golomb sequence $N=10^{4}$, and (f) the $\mathrm{CAN}(\mathrm{G})$ sequence $N=10^{4}$.

- Step 2) Compute the sequence $\left\{x_{n}\right\}_{n=1}^{N}$ that minimizes the criterion in (49) for $\left\{\boldsymbol{\alpha}_{p}\right\}_{p=1}^{2 N}$ fixed at their most recent values [see (58)].

- Iteration: repeat Steps 1) and 2) until a prespecified stop criterion is satisfied (see the CAP algorithm in Section II).

In the case of nonuniform weighting, we define the modified merit factor (MMF) using the weighted ISL as follows:

$$
\mathrm{MMF}=\frac{\left|r_{0}\right|^{2}}{2 \mathrm{WISL}}=\frac{N^{2}}{2 \sum_{k=1}^{N-1} w_{k}\left|r_{k}\right|^{2}} .
$$

We have observed empirically that WeCAN increases the MMF systematically when initialized by CAP, and vice versa. This motivates us to use CAP to initialize WeCAN, then use WeCAN to initialize CAP, and so on. The so-obtained combined iterative method is called WeCAN+CAP. As will be shown in Section V, when the maximum lag considered is smaller than half of the sequence length, WeCAN, CAP, and their combination WeCAN+CAP can generate sequences that have virtually an "infinite" MMF; the same is true even when the number of lags considered is smaller than half of the sequence length, provided the maximum lag under consideration is not too close to $N$ (see the next section for more details on this aspect).

\section{NUMERICAL EXAMPLES}

\section{A. ISL Design}

We compare the merit factors of the Golomb sequence ([12]), of the Frank sequence ([15]), and of the CAN sequence initial- 
ized by one of these two types of sequences [denoted as CAN $(\mathrm{G})$ and CAN(F), respectively]. A Golomb sequence $\{g(n)\}_{n=1}^{N}$ of length $N$ is defined as

$$
g(n)=e^{j \pi(n-1) n / N}, \quad n=1, \ldots, N
$$

where $N$ can be any positive integer. Frank sequences are only defined for lengths that are perfect squares. For $N=M^{2}$, a Frank sequence can be written as

$$
f(n M+k+1)=e^{j 2 \pi n k / M}, n, k=0,1, \ldots, M-1 .
$$

[Note that the above sequences can be easily computed for any value of $N$ of possible practical interest, with the only restriction that $N$ must be a perfect square in the case of (61).] We compute the merit factors of the above four types of sequences [Golomb, Frank, $\mathrm{CAN}(\mathrm{G})$ and $\mathrm{CAN}(\mathrm{F})]$ for the following lengths: $N=$ $3^{2}, 5^{2}, 10^{2}, 15^{2}, 20^{2}, 30^{2}, 70^{2}$, and $100^{2}$. The results are shown in Fig. 1 using a log-log scale. For all sequence lengths we consider, the $\mathrm{CAN}(\mathrm{G})$ and $\mathrm{CAN}(\mathrm{F})$ sequences give nearly the same merit factors; both are much larger than the merit factors given by the Golomb or Frank sequence. When $N=10^{4}$, the CAN $(\mathrm{G})$ sequence provides the largest merit factor of 1839.76 , which is more than ten times larger than that given by the Golomb sequence (which is 157.10). We also show the correlation levels of the Golomb and CAN $(\mathrm{G})$ sequences of lengths $N=10^{2}$, $10^{3}$, and $10^{4}$ in Fig. 2. The correlation level is defined as

$$
\text { correlation level }=20 \log _{10}\left|\frac{r_{k}}{r_{0}}\right|, \quad k=1, \ldots, N-1 .
$$

We note that the correlation sidelobes of the Golomb sequence are comparatively large for $k$ close to zero and $N-1$ (the same is true for the Frank sequence), while the $\operatorname{CAN}(\mathrm{G})$ sequence has relatively more uniform correlation sidelobes as $k$ increases from zero to $N-1$.

\section{B. WISL Design-A First Example}

Consider the design of a data sequence of length $N=100$. Suppose that we are interested in suppressing the correlations $r_{1}, \ldots, r_{25}$ and $r_{70}, \ldots, r_{79}$. Three methods are used to design the sequence. The first method is the original CA for (10), in which all correlation levels from $r_{1}$ to $r_{99}$ are taken into account. The second method is the CAP for (14), which focuses on $r_{1}, \ldots, r_{25}$ and $r_{70}, \ldots, r_{79}$ and therefore uses $P=80$, $Q=36$, and the following matrix $\mathbf{T}$ :

$$
\mathbf{T}=\left[\begin{array}{cc}
\mathbf{I}_{26} & 0 \\
0 & \vdots \\
\vdots & 0 \\
0 & \mathbf{I}_{10}
\end{array}\right]_{80 \times 36}
$$

where $\mathbf{I}_{K}$ denotes the $K \times K$ identity matrix. The third method is the WeCAN algorithm for (48) and (49), with the following weights used in the matrix $\Gamma$ in (41):

$$
\gamma_{k}=\left\{\begin{array}{ll}
1, & k \in[1,25] \cup[70,79] \\
0, & k \in[26,69] \cup[80,99]
\end{array} .\right.
$$

$\left[\gamma_{0}\right.$ is chosen to ensure the positive semi-definiteness of $\boldsymbol{\Gamma}$; more exactly, we choose $\gamma_{0}=12.05$ following the discussion right after (41).]

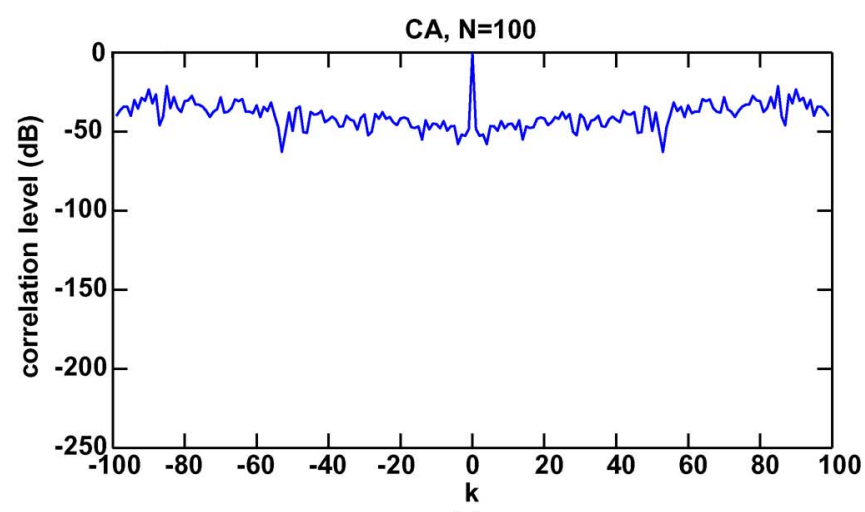

(a)

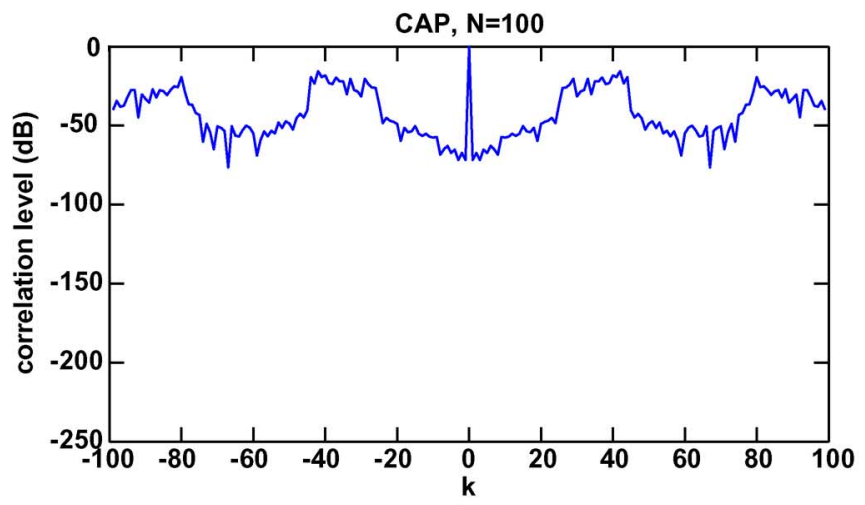

(b)

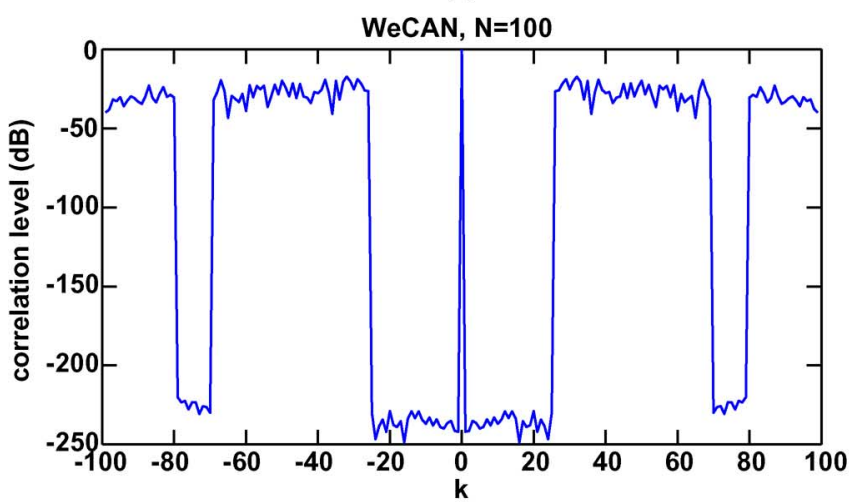

(c)

Fig. 3. Correlation levels of the CA, CAP, and WeCAN sequences of length $N=100$. (a) The CA sequence, (b) the CAP sequence, and (c) the WeCAN sequence designed under the WISL metric with weights in (65).

In this scenario, the MMF is as defined in (59) with

$$
w_{k}=\gamma_{k}^{2}=\left\{\begin{array}{ll}
1, & k \in[1,25] \cup[70,79] \\
0, & k \in[26,69] \cup[80,99]
\end{array} .\right.
$$

All three methods mentioned above are initialized by a randomly generated sequence [see Step 0) of the CAP algorithm in Section II]. The correlation levels of the designed sequences are shown in Fig. 3. The WeCAN sequence has correlation sidelobes that are practically zero at the required lags and that are much smaller than the sidelobes of the CA or CAP sequence and those of the Golomb or CAN $(\mathrm{G})$ sequence in the last subsection [see Fig. 2(a) and (b)]. Table I presents the corresponding MMF values. The MMF of the WeCAN sequence (which is practically infinite) is significantly larger than the other MMF values in the table. 


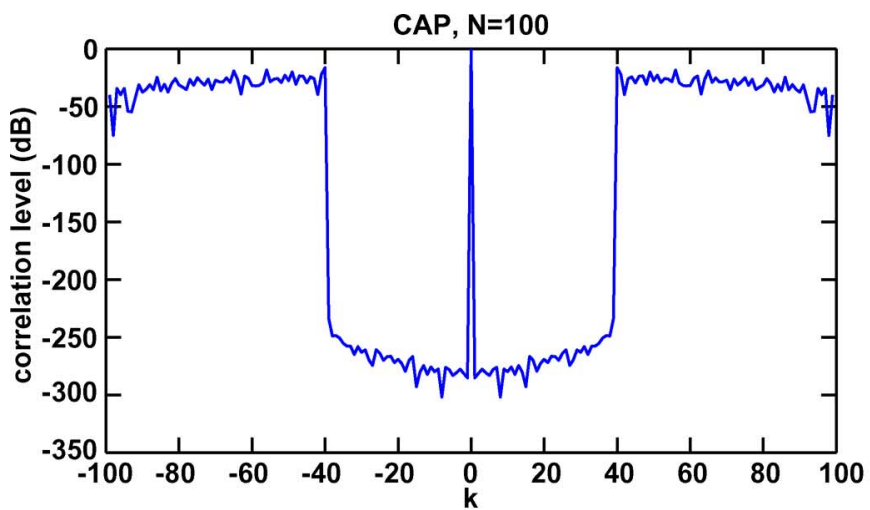

(a)

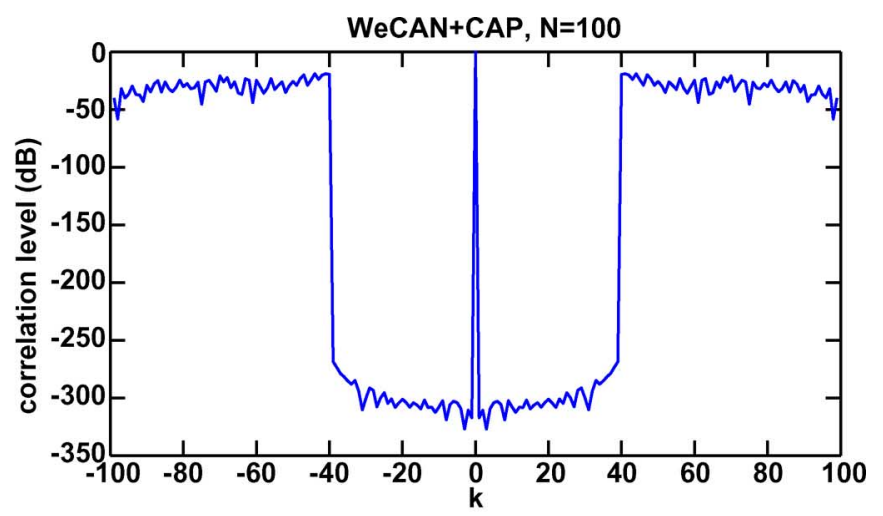

(b)

Fig. 4. Correlation levels of the CAP and WeCAN+CAP sequences of length $N=100$ designed under the WISL metric with weights in (66). (a) The CAP sequence and (b) the WeCAN+CAP sequence.

TABLE I

MMF VALUES FOR THE WEIGHTS IN (65) AND $N=100$

\begin{tabular}{|c|c|c|c|c|c|}
\hline & Golomb & CAN(G) & CA & CAP & WeCAN \\
\hline MMF & 32.55 & 142.64 & 68.07 & 229.02 & $1.06 \times 10^{21}$ \\
\hline
\end{tabular}

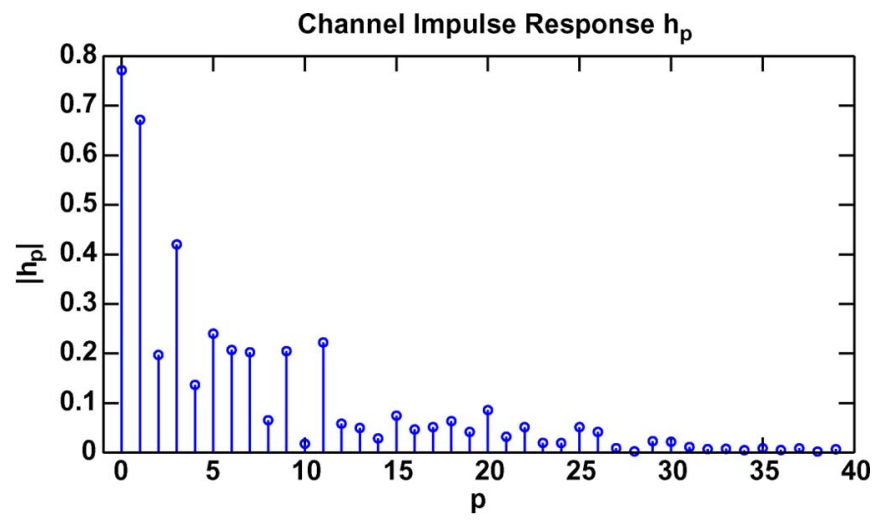

Fig. 5. Magnitude of the simulated channel impulse response $\mathbf{h}$.

TABLE II

MMF VALUES FOR THE WEIGHTS IN (66) AND $N=100$

\begin{tabular}{|c|c|c|c|}
\hline & CAN(G) & CAP & WeCAN+CAP \\
\hline MMF & 126.27 & $1.08 \times 10^{23}$ & $2.37 \times 10^{26}$ \\
\hline
\end{tabular}

The matrix $\tilde{\mathbf{X}}^{*} \tilde{\mathbf{X}}$ employed by CAP in this example [where $\tilde{\mathbf{X}}$ is given by (11)] is composed of $r_{1}, \ldots, r_{25}$ and $r_{70}, \ldots, r_{79}$ but also of $r_{45}, \ldots, r_{69}$. Therefore, although not of direct interest to us, $r_{45}, \ldots, r_{69}$ are minimized as well [see Fig. 3(b)], which increases the difficulty of the problem. If we consider fewer correlation lags (e.g., $r_{1}, \ldots, r_{9}$ and $r_{70}, \ldots, r_{79}$ only), CAP is also able to provide practically zero correlation sidelobes at the required lags. On the other hand, if more correlation lags are taken into account, then correlation sidelobes of either CAP or WeCAN become higher; the reason is that fewer and fewer degrees of freedom of the sequence $\left\{x_{n}\right\}_{n=1}^{N}$ can be used to control $\left|r_{k}\right|$ as $k$ increases beyond $N / 2$ (in particular, note that $\left|r_{N-1}\right|=1$ cannot be decreased).

\section{WISL Design-A Second Example}

Consider, once again, the design of a data sequence of length $N=100$ but now with the aim of suppressing the correlations $r_{1}, \ldots, r_{39}$. We compare the CAP sequence and the WeCAN+CAP sequence, both obtained using random initialization. The CAP sequence is generated using $P=Q=40$ and thus $\tilde{\mathbf{X}}=\overline{\mathbf{X}}$ in (11). The WeCAN+CAP sequence is generated as outlined at the end of Section IV. To construct the matrix $\Gamma$ in (41), we define

$$
\begin{aligned}
\gamma_{k} & = \begin{cases}1, & k \in[1,39] \\
0, & k \in[40,99]\end{cases} \\
w_{k} & =\gamma_{k}^{2}, \quad k=1, \ldots, 99
\end{aligned}
$$

and choose $\gamma_{0}$ such that $\Gamma \geq 0$.

Fig. 4 shows the correlation levels of the so-obtained CAP and WeCAN+CAP sequences, and Table II presents the corresponding MMF values [the $\mathrm{CAN}(\mathrm{G})$ sequence is included in the table for the sake of comparison]. Both sequences have practically zero correlation sidelobes from $r_{1}$ to $r_{P-1}$, and the corresponding MMF can be considered to be infinity (the smallest correlation level in Fig. 4 is around -320 dB, i.e., $10^{-16}$, which is the smallest number that can be properly handled in MATLAB and can thus be considered as "zero").

A point worth mentioning here is that the CAP and WeCAN+CAP algorithms are able to provide an "infinite" MMF in this example if $P \leq 50$. The reason is that the number of degrees of freedom in this example is $N-1=99$ (there are $N-1$ free phases as the initial phase does not matter) and our goal is to match $2(P-1)$ real numbers (i.e., the real and imaginary parts of $\left.r_{1}, \ldots, r_{P-1}\right)$. Consequently the matching is possible in principle only when $2(P-1) \leq N-1$, which leads to $P \leq(N+1) / 2$. In the next subsection, $P$ is fixed to 40 and $N$ is varied from 100 to 500 , in which case the CAP or WeCAN+CAP algorithm consistently generates sequences that have an "infinite" MMF.

Remark: The WeCAN algorithm is also able to provide an "infinite" MMF in this example, although we do not show its results here for brevity. Another fact worth pointing out is that both CAP and WeCAN algorithms require a proper value of the stop criterion parameter $\epsilon$ (see Section II) to perform well. When the number of considered correlation lags is less than $(N+1) / 2$ and $N$ is relatively small (such as $N \sim 10^{2}$ ), a sufficiently small $\epsilon$ should be used (e.g., $\epsilon=10^{-13}$ in the examples in this and the last subsection) to permit enough many iterations, which drive the correlation sidelobes to zero, to be run; in other cases, a "moderate" $\epsilon$ (depending on the application, such as $10^{-5}$ ) 


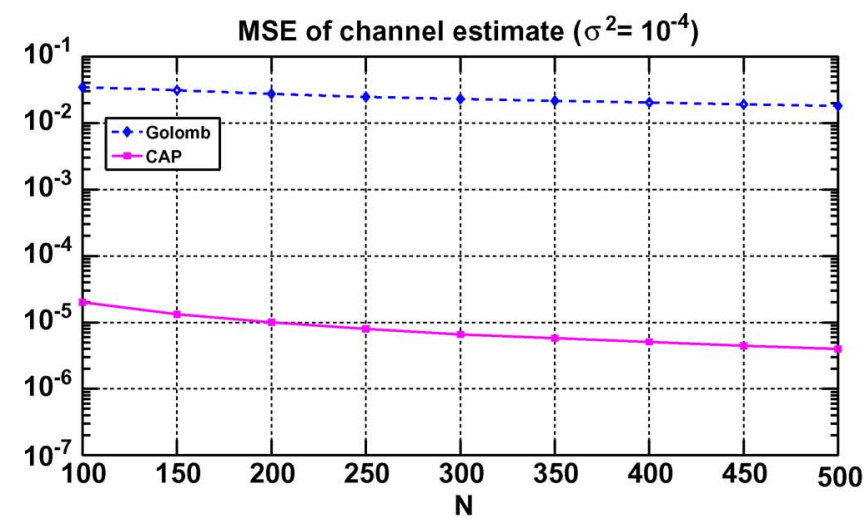

(a)

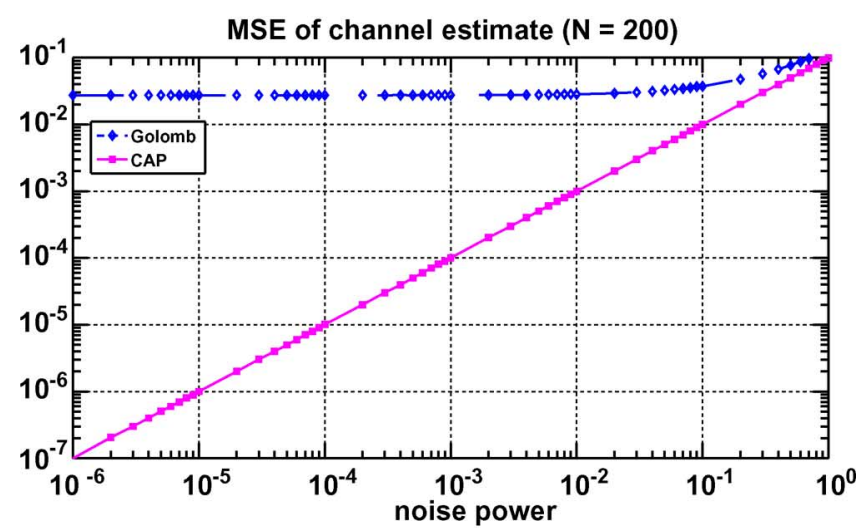

(b)

Fig. 6. The MSE of the estimated $\hat{\mathbf{h}}$ using two training sequences: the Golomb sequence and the CAP sequence. (a) The noise power $\sigma^{2}$ is fixed at $10^{-4}$ and the sequence length $N$ is varied from 100 to 500 . (b) $N$ is fixed at 200 and $\sigma^{2}$ is varied from $10^{-6}$ to one.

is preferable to prevent the program from running indefinitely without suppressing the correlation sidelobes anymore. In contrast with this, WeCAN+CAP is quite insensitive to the choice of $\epsilon\left(\epsilon=10^{-5}\right.$ is appropriate for WeCAN+CAP in all cases that we have tested) and it outperforms CAP in terms of MMF, especially for large values of $N$.

\section{FIR Channel Estimation}

Consider a channel with finite impulse response (FIR) channel impulse response $\left\{h_{p}\right\}_{p=0}^{P-1}$ whose estimation is our main goal (the number of channel taps $P$ is assumed to be known). Suppose we transmit a probing sequence $\left\{x_{n}\right\}_{n=1}^{N}$ and obtain the received signal

$$
y_{n}=\sum_{p=0}^{P-1} h_{p} x_{n-p}+e_{n}, \quad n=1, \ldots, N+P-1
$$

where $\left\{e_{n}\right\}_{n=1}^{N+P-1}$ is an independent identically distributed complex Gaussian white noise sequence with zero mean and variance $\sigma^{2}$. Equation (67) can be written in the following more compact form:

$$
\mathbf{y}=\overline{\mathbf{X}} \mathbf{h}+\mathbf{e}
$$

where $\overline{\mathbf{X}}$ is as defined in (12) and

$$
\begin{aligned}
& \mathbf{y}=\left[\begin{array}{lll}
y_{1} & \cdots & y_{N+P-1}
\end{array}\right]^{T}, \quad \mathbf{h}=\left[\begin{array}{lll}
h_{0} & \cdots & h_{P-1}
\end{array}\right]^{T} \\
& \mathbf{e}=\left[\begin{array}{lll}
e_{1} & \cdots & e_{N+P-1}
\end{array}\right]^{T} .
\end{aligned}
$$

Let $\overline{\mathbf{x}}_{p}$ denote the $p$ th column of the matrix $\overline{\mathbf{X}}$. We use $\overline{\mathbf{x}}_{p}$ as a "matched filter" to determine $h_{p}$ from $\mathbf{y}$, which leads to the following estimate of $h_{p}$ :

$$
\hat{h}_{p}=\frac{1}{N} \overline{\mathbf{x}}_{p}^{*} \mathbf{y} .
$$

Let the number of channel taps be $P=40$. Fig. 5 shows the magnitude of the simulated channel impulse response $\left\{\left|h_{p}\right|\right\}_{p=0}^{P-1}$. We perform two experiments to compare the Golomb sequence and the CAP sequence. In one experiment, the noise power $\sigma^{2}$ is fixed at $10^{-4}$ and the sequence length $N$ is varied from 100 to 500. In the other experiment, $N$ is fixed at 200 and $\sigma^{2}$ is varied from $10^{-6}$ to one. For each pair $\left(N, \sigma^{2}\right), 500$ Monte Carlo trials are run (in which the noise sequence $\mathbf{e}$ is varied) and the mean-squared error (MSE) of $\hat{\mathbf{h}}$ is recorded. Fig. 6 shows the MSE of $\hat{\mathbf{h}}$ in the two situations. Due to better autocorrelation properties, the CAP sequence generates consistently smaller MSE than the Golomb sequence. In particular, it is interesting to observe from Fig. 6(b) that as $\sigma^{2}$ decreases, the MSE of $\hat{\mathbf{h}}$ corresponding to the CAP sequence is decreasing linearly (and becomes zero as $\sigma^{2}$ goes to zero), while the performance of the Golomb sequence is limited to a certain level because of its nonzero correlation sidelobes, which induce an estimation bias.

\section{CONCLUDING REMARKS}

We have presented several cyclic algorithms-CAP, CAN, WeCAN, and WeCAN+CAP — which can be used to design unimodular sequences that have good autocorrelation properties. CAN can be used to design very long sequences (of length $N$ up to $10^{6}$ ), a design problem that can hardly be handled by other algorithms proposed in the previous literature. CAN deals with the ISL metric, i.e., it considers all unweighted correlation lags from $r_{1}$ up to $r_{N-1}$, whereas CAP, WeCAN, and WeCAN+CAP aim to minimize weighted-ISL metrics. We have shown that, in particular, the latter three algorithms can be used to design sequences that have virtually zero autocorrelation sidelobes in a specified lag interval. CAP, WeCAN, and WeCAN+CAP can be used to design sequences of lengths $N \sim 10^{3}$ or larger, depending on how many lags are considered. A number of numerical examples have been provided to demonstrate the good autocorrelation properties of the unimodular sequences designed using the proposed algorithms.

\section{REFERENCES}

[1] J. Jedwab, "A survey of the merit factor problem for binary sequences," in Sequences and Their Applications-SETA 2004, T. Helleseth, D. Sarwate, H. Y. Song, and K. Yang, Eds. Heidelberg, Germany: Springer-Verlag, 2005, vol. 3486, Lecture Notes in Computer Science, pp. $30-55$.

[2] T. Høholdt, "The merit factor problem for binary sequences," in Applied Algebra, Algebraic Algorithms and Error-Correcting Codes, M. Fossorier, H. Imai, S. Lin, and A. Poli, Eds. Heidelberg, Germany: Springer-Verlag, 2006, vol. 3857, Lecture Notes in Computer Science, pp. 51-59.

[3] M. J. E. Golay, "Sieves for low autocorrelation binary sequences," IEEE Trans. Inf. Theory, vol. IT-23, pp. 43-51, Jan. 1977.

[4] J. Lindner, "Binary sequences up to length 40 with best possible autocorrelation function," Electron. Lett., vol. 11, no. 21, pp. 507-507, Oct. 1975. 
[5] C. D. Groot, D. Würtz, and K. H. Hoffmann, "Low autocorrelation binary sequences: Exact enumeration and optimization by evolutionary strategies," Optimization, vol. 23, no. 4, pp. 369-384, 1992.

[6] S. Mertens, "Exhaustive search for low-autocorrelation binary sequences," J. Phys. A, vol. 29, pp. 473-481, 1996.

[7] R. Ferguson and J. Knauer, "Optimization methods for binary sequences-The merit factor problem," in MITACS 6th Annu. Conf., Calgary, AB, Canada, May 2005, Univ. of Calgary.

[8] H. D. Schotten and H. D. Lüke, "On the search for low correlated binary sequences," Int. J. Electron. Commun., vol. 59, no. 2, pp. 67-78, 2005.

[9] J. Jedwab, "What can be used instead of a Barker sequence?," Contemp. Math, 2008.

[10] T. Xiong and J. Hall, "Construction of even length binary sequences with asymptotic merit factor 6," IEEE Trans. Inf. Theory, vol. 54, pp. 931-935, Feb. 2008.

[11] P. B. Rapajic and R. A. Kennedy, "Merit factor based comparison of new polyphase sequences," IEEE Commun. Lett., vol. 2, pp. 269-270, Oct. 1998.

[12] N. Zhang and S. W. Golomb, "Polyphase sequence with low autocorrelations," IEEE Trans. Inf. Theory, vol. 39, pp. 1085-1089, May 1993.

[13] P. Borwein and R. Ferguson, "Polyphase sequences with low autocorrelation,” IEEE Trans. Inf. Theory, vol. 51, pp. 1564-1567, Apr. 2005.

[14] U. Somaini and M. Ackroyd, "Uniform complex codes with low autocorrelation sidelobes (corresp.)," IEEE Trans. Inf. Theory, vol. IT-20, pp. 689-691, Sep. 1974.

[15] R. Frank, "Polyphase codes with good nonperiodic correlation properties," IEEE Trans. Inf. Theory, vol. IT-9, pp. 43-45, Jan. 1963.

[16] F. Kretschmer, Jr. and K. Gerlach, "Low sidelobe radar waveforms derived from orthogonal matrices," IEEE Trans. Aerosp. Electron. Syst., vol. 27, pp. 92-102, Jan. 1991.

[17] H. A. Khan, Y. Zhang, C. Ji, C. J. Stevens, D. J. Edwards, and D. O'Brien, "Optimizing polyphase sequences for orthogonal netted radar," IEEE Signal Process. Lett., vol. 13, pp. 589-592, Oct. 2006.

[18] J. Benedetto and J. Donatelli, "Ambiguity function and frame-theoretic properties of periodic zero-autocorrelation waveforms," IEEE J. Sel. Topics Signal Process., vol. 1, pp. 6-20, Jun. 2007.

[19] P. Stoica, J. Li, and X. Zhu, "Waveform synthesis for diversity-based transmit beampattern design," IEEE Trans. Signal Process., vol. 56, pp. 2593-2598, Jun. 2008.

[20] J. A. Tropp, I. S. Dhillon, R. W. Heath, and T. Strohmer, "Designing structured tight frames via an alternating projection method," IEEE Trans. Inf. Theory, vol. 51, pp. 188-209, Jan. 2005.

[21] J. Li, P. Stoica, and X. Zheng, "Signal synthesis and receiver design for MIMO radar imaging," IEEE Trans. Signal Process., vol. 56, pp. 3959-3968, Aug. 2008.

[22] H. He, P. Stoica, and J. Li, "Designing unimodular sequence sets with good correlations-Including an application to MIMO radar," IEEE Trans. Signal Process., Jan. 2009 [Online]. Available: http://plaza.ufl. edu/haohe/papers/draft HeStoicaLi2009Jan.pdf, submitted for publication

[23] J. Ling, T. Yardibi, X. Su, H. He, and J. Li, "Enhanced channel estimation and symbol detection for high speed MIMO underwater acoustic communications," in Proc. IEEE 13th DSP Workshop 5th SPE Workshop, Marco Island, FL, Jan. 2009.

[24] P. Stoica and R. L. Moses, Spectral Analysis of Signals. Upper Saddle River, NJ: Prentice-Hall, 2005.

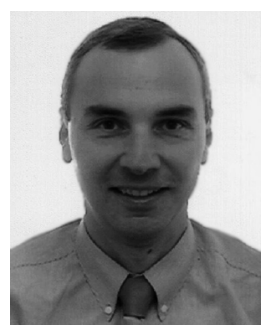

Petre Stoica (F'94) received the D.Sc. degree in automatic control from the Polytechnic Institute of Bucharest (BPI), Bucharest, Romania, in 1979 and the honorary doctorate degree in science from Uppsala University (UU), Uppsala, Sweden, in 1993.

$\mathrm{He}$ is a Professor of systems modeling with the Division of Systems and Control, Department of Information Technology, UU. Previously, he was a Professor of system identification and signal processing with the Faculty of Automatic Control and Computers, BPI. He held longer visiting positions with Eindhoven University of Technology, Eindhoven, The Netherlands; Chalmers University of Technology, Gothenburg, Sweden (where he held a Jubilee Visiting Professorship); UU; the University of Florida, Gainesville; and
Stanford University, Stanford, CA. His main scientific interests are in the areas of system identification, time-series analysis and prediction, statistical signal and array processing, spectral analysis, wireless communications, and radar signal processing. He has published nine books, ten book chapters, and some 500 papers in archival journals and conference records. His most recent book is (with R. Moses) Spectral Analysis of Signals (Englewood Cliffs, NJ: Prentice-Hall, 2005). He is on the editorial boards of six journals. He was a co-Guest Editor for several special issues on system identification, signal processing, spectral analysis, and radar for some of the aforementioned journals, as well as for the Proceedings of the Institution of Electrical Engineers. He has been a member of the international program committees of many topical conferences.

Dr. Stoica is a Fellow of the Royal Statistical Society, a member of the Royal Swedish Academy of Engineering Sciences, and an honorary member of the Romanian Academy. From 1981 to 1986, he was a Director of the International Time-Series Analysis and Forecasting Society. He was a member of the IFAC Technical Committee on Modeling, Identification, and Signal Processing. $\mathrm{He}$ was a corecipient of the IEEE ASSP Senior Award for a paper on statistical aspects of array signal processing. He also received the Technical Achievement Award from the IEEE Signal Processing Society. In 1998, he received a Senior Individual Grant Award from the Swedish Foundation for Strategic Research. He was a corecipient of the 1998 EURASIP Best Paper Award for Signal Processing for a work on parameter estimation of exponential signals with time-varying amplitude, a 1999 IEEE Signal Processing Society Best Paper Award for a paper on parameter and rank estimation of reduced-rank regression, a 2000 IEEE Third Millennium Medal, and the 2000 W. R. G. Baker Prize Paper Award for a paper on maximum likelihood methods for radar.

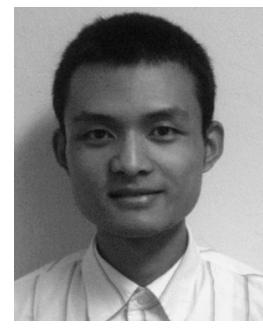

Hao He (S'08) received the B.Sc. degree in electrical engineering from the University of Science and Technology of China (USTC), Hefei, in 2007. $\mathrm{He}$ is currently pursuing the Ph.D. degree with the Department of Electrical Engineering, University of Florida, Gainesville.

His research interests are in the areas of spectral estimation and radar signal processing.

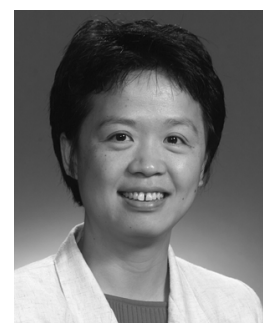

Jian Li (S'87-M'91-SM'97-F'05) received the M.Sc. and Ph.D. degrees in electrical engineering from The Ohio State University (OSU), Columbus, in 1987 and 1991, respectively.

From April to June 1991, she was an Adjunct Assistant Professor with the Department of Electrical Engineering, OSU. From July 1991 to June 1993, she was an Assistant Professor with the Department of Electrical Engineering, University of Kentucky, Lexington. Since August 1993, she has been with the Department of Electrical and Computer Engineering, University of Florida, Gainesville, where she is currently a Professor. In Fal 2007, she was on sabbatical leave at the Massachusetts Institute of Technology, Cambridge. Her current research interests include spectral estimation, statistical and array signal processing, and their applications. She was a member of the Editorial Board of Signal Processing from 2005 to 2007. She has been a member of the Editorial Board of Digital Signal Processing - A Review Journal since 2006.

Dr. Li is a Fellow of IET and a member of Sigma Xi and Phi Kappa Phi. She received the 1994 National Science Foundation Young Investigator Award and the 1996 Office of Naval Research Young Investigator Award. She was an Executive Committee Member of the 2002 International Conference on Acoustics, Speech, and Signal Processing, Orlando, FL, in May 2002. She was an Associate Editor of the IEEE TRANSACTIONS ON SIGNAL PROCESSING from 1999 to 2005 and an Associate Editor of the IEEE SIGNAL PROCESSING MAGAZINE from 2003 to 2005. She is presently a member of the Sensor Array and Multichannel Technical Committee, IEEE Signal Processing Society. She is a coauthor of papers that received the First and Second Place Best Student Paper Awards, respectively, at the 2005 and 2007 Annual Asilomar Conferences on Signals, Systems, and Computers, Pacific Grove, CA. She is also a coauthor of the paper that received the M. Barry Carlton Award for the best paper published in IEEE TRANSACTIONS ON AEROSPACE AND ELECTRONIC SYSTEMS in 2005. 